\title{
Solubility and Anisotropic Migration Behaviors of Helium in bec Iron Under Strain
}

\author{
Yue $\mathrm{Yu}^{1} \cdot$ Ben $\mathrm{Xu}^{1} \cdot$ Hao Chen ${ }^{1} \cdot$ Zhi-Gang Yang ${ }^{1} \cdot$ Chi Zhang $^{1}$
}

Received: 31 January 2017/Revised: 4 April 2017/Published online: 9 May 2017

(C) The Chinese Society for Metals and Springer-Verlag Berlin Heidelberg 2017

\begin{abstract}
The different solution and migration behaviors of tetrahedral and octahedral interstitial helium in bcc iron have been investigated by using first principles calculations. We showed that the tetrahedral site has less charge transfer and less redistribution of the density of states but stronger bonding and a lower solution energy. This is due to the coupling between the symmetrical facts of the two interstitial atoms and the $3 d$ orbitals of Fe atoms. The solution energies of both sites are not significantly influenced by applied normal strains of $2 \%$ and $4 \%$. In contrast, the migration barriers have the reverse trends for different migration directions under strain, which can be explained by an anisotropic elastic energy change and charge transfer. The lower migration energy along certain directions under strain can facilitate the segregation of helium and the formation of helium bubbles.
\end{abstract}

\section{KEY WORDS: Strain; Point defects; Migration; Density functional theory (DFT); Iron; Helium embrittlement}

\section{Introduction}

The stability and reliability of structural materials are of crucial importance to the commercial use of fusion reactors. As one of the most promising structural materials used in the International Thermonuclear Experimental Reactor (ITER) Test Blanket Module (TBM), reduced activation ferritic or martensitic steel (RAFM steel) has been the focus of experimental and simulation investigations [1-4].

Helium embrittlement is a severe problem that degrades the mechanical properties of RAFM steel. According to the ITER design, the RAFM steel used in the TBM is adversely

Available online at http://link.springer.com/journal/40195.

\section{Ben Xu}

xuben@mail.tsinghua.edu.cn

1 Key Laboratory of Advanced Materials of Ministry of Education, School of Materials Science and Engineering, Tsinghua University, Beijing 100084, China affected by the high-flux neutron ( $14 \mathrm{MeV}$ ) produced from the deuterium-tritium reaction, which induces transmutation in the lattice, releasing helium and hydrogen. The single helium atoms that enter the lattice will first dissolve and then migrate and interact with the irradiation-induced defects [5-7]. The subsequent migration and coalescence of helium and helium-vacancy clusters can lead to the formation of helium bubbles, voids and blisters [5, 8], which further evolves into void swelling, surface roughening and high-temperature granular embrittlement thus deteriorates the mechanical properties of the steel [9-12].

The complicated service conditions of fusion reactor make it difficult to interpret the mechanisms of helium embrittlement. High-flux magnetic fields, high stress and periodically changing thermal stresses (caused by the periodic discharge of the high-temperature plasma) $[3,13-15]$ might superimpose with the degradation effect of the helium. Previous experiments have evidenced that the growth of helium bubbles is accelerated by tensile strain [16], which means that it is necessary to consider the effect of stress on the helium behaviors. 
To fully understand the helium embrittlement and predict the mechanical behaviors of RAFM steel under service conditions such as strain, atomistic studies tracing back to the solution and migration behaviors of single helium atoms are important. Although various experiments have evidenced the changes caused by helium in the microstructure of iron [10, 16-18], developing an experimental explanation from the atomistic scale is extremely difficult. In contrast, simulation from different scales can provide useful information on how the microstructure evolves from single helium atoms to helium clusters and bubbles [19-22].

First principles calculations, due to its accurate description of electronic interactions, have been widely used to study the solution and migration of single helium atom. Energetic data of these elementary processes have been obtained using density functional theory (DFT) calculations. Previous DFT calculations [20, 23, 24] predicted that the tetrahedral configuration is more stable than the octahedral one by $0.2 \mathrm{eV}$. The migration energy has also been calculated by using the drag method [20] or static calculation of the saddle point energy [25]. However, the physics for the energy difference between the tetrahedral and octahedral sites has not been completely revealed yet.

To understand the helium bubble growth under strain at the microscopic scale, our research focused on the solution energy and migration barriers under different strains, and the electronic structure and charge transfer which have important relations with the solution and migration energies.

\section{Simulation Method}

The calculations were performed by using the Vienna ab initio Simulation Package (VASP) and were achieved in the framework of Blöchl's projector augmented wave (PAW) method [26, 27] within the generalized gradient approximation (GGA) proposed by Perdew, Burke and Ernzerhof [28]. To simulate the point defects in the matrix, a supercell model was built up with periodic boundary conditions, and the Monkhorst and Pack scheme was used to perform the Brillion zone sampling. After performing a series of parameter optimizations, an $8 \times 8 \times 8 \mathrm{k}$-point mesh was chosen, and the cutoff energy was set to $520 \mathrm{eV}$ for $2 \times 2 \times 2$ supercell lattice containing one helium atom. The calculations were performed with a spin-polarization, and initial magnetic moments were also specified for each atom. The equilibrium lattice parameter for the pure $\mathrm{Fe} 2 \times 2 \times 2$ supercell was $5.665 \AA$. The energy minimization continued until the variation of the total energy converges to $10^{-5} \mathrm{eV}$ and the forces on all the atoms converge to less than $10^{-3} \mathrm{eV} / \AA$. When a helium atom was put into the lattice, the lattice was allowed to expand to reach the equilibrium state, which provided information on the stress and strain induced by a certain concentration of helium.

The charge transfer of the interstitial sites was analyzed by using charge density difference analysis and Bader charge analysis [29-31], and the result was compared with the density of states (DOS) to show the relations between the charge transfer and bonding. The solution energy was used to check the stability of the interstitial helium atoms, which was determined using the formula:

$E_{\mathrm{sol}}=E(N \mathrm{Fe}+\mathrm{He})-E(N \mathrm{Fe})-E\left(\mathrm{He}_{\text {isolated }}\right)$,

and the solution energy was further divided into the deformation part and electronic part for better understanding.

The migration energy barrier of helium moving from one stable tetrahedral interstitial to the neighboring tetrahedral interstitial was calculated by using the climbingimage nudged elastic band (CI-NEB) method [32, 33], where the two neighboring interstitial sites are set as the starting and ending states, respectively.

The strain was applied uniaxially in this research, by fixing the boundary in one direction and relaxing in the other two directions. The formula for calculating the strain then should be changed to $E_{\varepsilon}^{\mathrm{sol}}=E_{\varepsilon, \mathrm{He}}-E_{\varepsilon}-E_{\mathrm{He}}$, with $E_{\varepsilon, \mathrm{He}}$ and $E_{\varepsilon}$ being the total energy under strain $\varepsilon$ with and without helium and $E_{\mathrm{He}}$ being the energy of a single helium atom.

\section{Results and Discussion}

\subsection{Solution Energy}

The solution energies were calculated for the tetrahedral and octahedral interstitial sites and verified by the analysis of the charge transfer and DOS. The lower energy of the tetrahedral site suggests it to be the more stable site, which is consistent with other reported results, as listed in Table 1.

The solution energy was further divided into an elastic part $E_{\mathrm{d}}$ and an electronic part $E_{\mathrm{e}} . E_{\mathrm{d}}$ is the variation of total energy caused by introducing one helium atom into the supercell, which follows the equation below using elastic theory:

$$
\begin{aligned}
E_{\mathrm{d}} & =E\left(V,\left\{e_{i}\right\}\right)-E\left(V_{0}, 0\right) \\
& =V_{0} \sum_{i=1}^{6} \sigma_{i} e_{i}+\frac{V_{0}}{2} \sum_{i, j=1}^{6} C_{i j} e_{i} e_{j}+\ldots,
\end{aligned}
$$

where $V_{0}$ is the volume of the supercell without strain, and $\left\{e_{i}\right\}$ and $\left\{\sigma_{i}\right\}$ are the strain and stress caused by the 
Table 1 Solution energies of tetrahedral and octahedral interstitial sites in a $2 \times 2 \times 2$ supercell (without strain)

\begin{tabular}{lllll}
\hline$E_{\text {sol }}(\mathrm{eV})$ & Our calculation & Literature values [23] by VASP & Literature values [24] by VASP & Literature values [20] by SIESTA \\
\hline Tetrahedral site & 4.46 & 4.37 & 4.56 & 4.38 \\
Octahedral site & 4.65 & 4.60 & 4.76 & 4.55 \\
\hline
\end{tabular}

Table 2 Strain induced by helium in supercells with different interstitials

\begin{tabular}{llll}
\hline Interstitial site & $x(\%)$ & $y(\%)$ & $z(\%)$ \\
\hline Tetrahedral & 1.65 & 1.65 & 2.04 \\
Octahedral & 1.08 & 1.08 & 3.29 \\
\hline
\end{tabular}

Positive values stand for expansion of the lattice and vice versa

Table 3 Stress induced by helium in supercells with different interstitials

\begin{tabular}{llll}
\hline Interstitial site & $x(\mathrm{GPa})$ & $y(\mathrm{GPa})$ & $z(\mathrm{GPa})$ \\
\hline Tetrahedral & 9.95 & 9.95 & 10.3 \\
Octahedral & 9.62 & 9.62 & 11.6 \\
\hline
\end{tabular}

Positive values represent compressive stress, and negative values represent tensile stress

Table 4 Elastic part and electronic part of the interstitial solution energy

\begin{tabular}{lll}
\hline Interstitial site & Tetrahedral & Octahedral \\
\hline$E_{\text {sol }}(\mathrm{eV})$ & 4.46 & 4.65 \\
$E_{\mathrm{d}}(\mathrm{eV})$ & 0.92 & 1.01 \\
$\mathrm{E}_{\mathrm{e}}(\mathrm{eV})$ & 3.54 & 3.64 \\
\hline
\end{tabular}

interstitial helium, which are listed in Tables 2 and 3, respectively. The elastic constants of the cubic system, $C_{11}=261 \mathrm{GPa}$ and $C_{12}=154 \mathrm{GPa}$, are calculated by VASP. Then, $E_{\mathrm{d}}$ was calculated by using the equation above while neglecting the higher order terms, and $E_{\mathrm{e}}$ was directly calculated by subtracting $E_{\mathrm{d}}$ from $E_{\text {sol }}$. The results are listed in Table 4. Compared with the octahedral sites, the tetrahedral site occupation causes both smaller elastic and electronic solution energies.

The solution energy under a certain strain was also calculated, as listed in Table 5. It should be noted that the solution energy of both the tetrahedral sites and octahedral sites only changes a little when the lattice is strained, showing a slight decrease with both the tensile and compressive strains. Helium is more stable at the tetrahedral interstitial sites, no matter how the strain condition changes.
Table 5 Solution energies of $\mathrm{T}$ and $\mathrm{O}$ sites for different strains applied

\begin{tabular}{lll}
\hline Strain & $E_{\text {sol }}^{\text {tetra }}(\mathrm{eV})$ & $E_{\text {sol }}^{\text {octa }}(\mathrm{eV})$ \\
\hline$-4 \%$ (compressive) & 4.433 & 4.629 \\
$-2 \%$ (compressive) & 4.456 & 4.649 \\
0 (none) & 4.458 & 4.652 \\
$2 \%$ (tensile) & 4.446 & 4.650 \\
$4 \%$ (tensile) & 4.447 & 4.639 \\
\hline
\end{tabular}

The strain direction is [100]

\subsection{Charge Transfer and Bonds}

To further investigate the electronic part of the solution energy, the bonds were analyzed by the charge density difference, Bader charge analysis and DOS. The charge density difference was calculated for both the octa- and tetra-sites, and the cross section of the surface that contains helium and its nearest iron atoms is plotted in Fig. 1. The charge density is polarized for both the tetrahedral and octahedral sites, and the center of the positive charge is clearly located near the position of the helium, which indicates the ionic bonding between the helium and iron atoms. A comparison of Fig. 1a with c shows fewer contour lines, both above and below 0 , for the tetrahedral site, which suggests that the charge density has less change around the tetrahedral interstitial than the octahedral interstitial site.

Detailed information about the charge transfer is listed in Table 6 for both the tetrahedral and octahedral interstitial sites from the Bader charge analysis. In the case of the tetrahedral interstitial, the Bader charge for the first nearest neighboring $\mathrm{Fe}$ atom $\left(\mathrm{Fe}_{1 \mathrm{nn}}\right)$ slightly decreases by less than $0.05 \mathrm{e}$, while that for the interstitial $\mathrm{He}$ atom increases by 0.18 e. For the octahedral interstitial, $\mathrm{Fe}_{1 \mathrm{nn}}$ loses 0.15 e, and the interstitial $\mathrm{He}$ increases by $0.21 \mathrm{e}$. Clearly, there are more charge transfers between the octahedral helium and the iron atoms around it, and the interstitial helium gains electrons, corresponding to the results of the charge density analysis. However, because of the different symmetries of octahedral and tetrahedral interstitial sites, more charge transfer does not necessarily result in stronger bonding.

The DOS was then plotted to understand the bonding. The total densities of states (t-DOSs) of the interstitial 
(a)

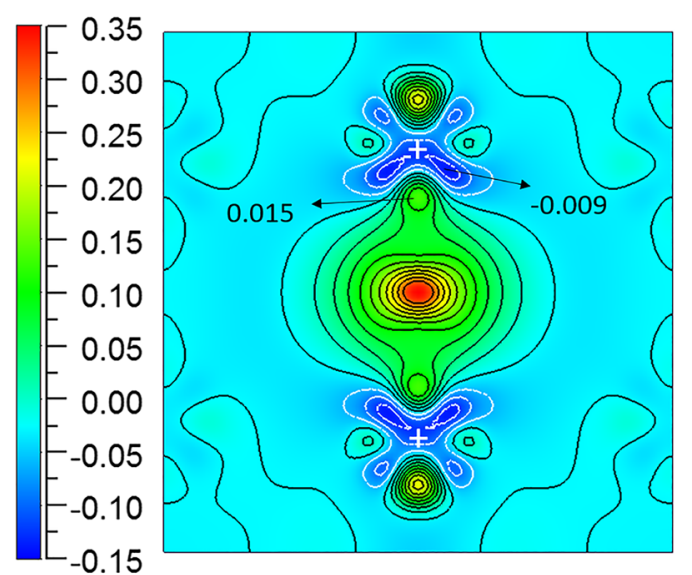

(c)

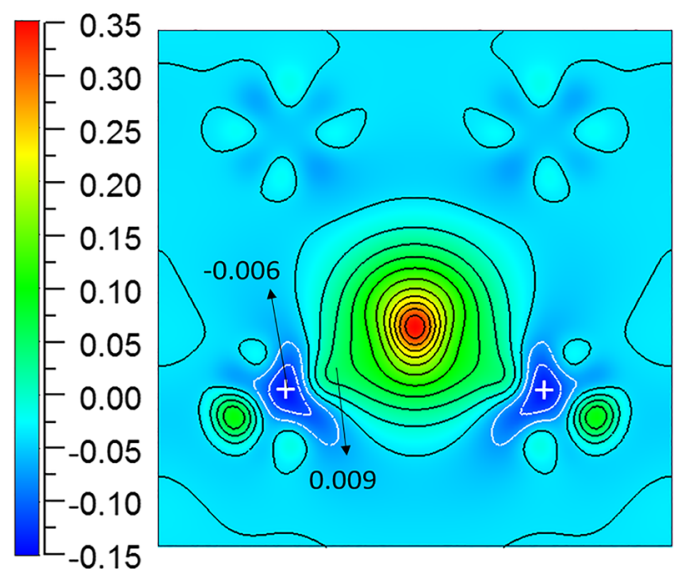

(b)

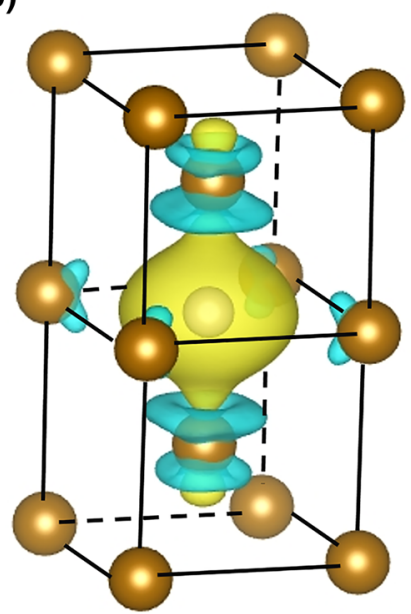

(d)

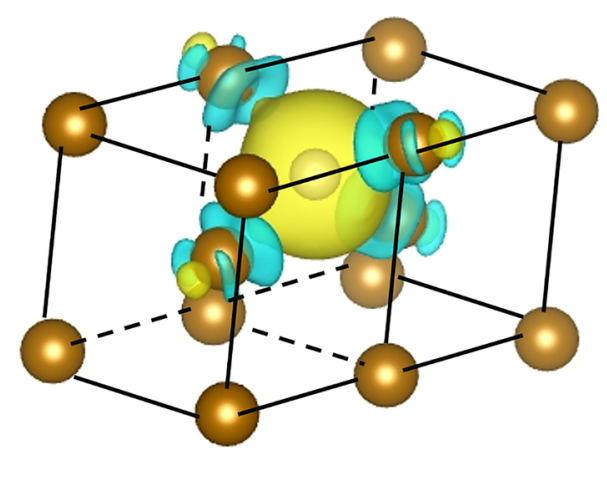

Fig. 1 Charge density difference around interstitial helium atom and its nearest iron atoms: a, b charge density difference of the octahedral site; c, d charge density difference of the tetrahedral site. a, c Cross-sectional surface containing the helium atom and the nearest iron atoms corresponding to $\mathbf{b}, \mathbf{d}$, respectively. The color red means the highest charge density difference, while blue means the lowest. The contour surfaces above 0 are shown by black lines separated by $0.003 \mathrm{eV} / \AA^{3}$, while those below 0 are shown by white lines. In $\mathbf{c}$, d, the blue part suggests a loss of charge density, while yellow suggests an increase in charge density. Helium lies in the center of the red part, while the positions of the iron atoms are shown by the white crosses

Table 6 Bader charge analysis for Fe and He atoms in a $2 \times 2 \times 2$ lattice. $\left(\mathrm{e}=1.6 \times 10^{-19} \mathrm{C}\right)$

\begin{tabular}{|c|c|c|}
\hline & Tetrahedral & Octahedral \\
\hline Atom & Bader charge (e) & Bader charge (e) \\
\hline $\mathrm{Fe}_{1 \mathrm{nn}}$ & 13.9552 & 13.8575 \\
\hline $\mathrm{He}$ & 2.1819 & 2.2142 \\
\hline $\mathrm{Fe}_{2 \mathrm{nn}}$ & 13.9906 & 13.9547 \\
\hline Bond & Bond distance $(\AA)$ & Bond distance $(\AA)$ \\
\hline $\mathrm{Fe}_{1 \mathrm{nn}}-\mathrm{He}$ & 1.6367 & 1.5021 \\
\hline $\mathrm{Fe}_{2 \mathrm{nn}}-\mathrm{He}$ & 2.3146 & 2.1243 \\
\hline
\end{tabular}

helium and its nearest iron atom are plotted in Fig. 2. For the t-DOS of helium, the highest peak for spin-up channel of the tetrahedral site (at $0.18 \mathrm{eV}$ ) is higher than that of the octahedral site (at $-1.49 \mathrm{eV}$ ), and the highest peak for spin-down channel of the tetrahedral site (at $2.29 \mathrm{eV}$ ) is also higher than that of the octahedral site (at $1.81 \mathrm{eV}$ ). Besides these peaks, the DOSs of these two sites are similar. Considering that the t-DOS of helium is much smaller than that of iron, a higher t-DOS of helium means more overlap with the t-DOS of iron at a tetrahedral site, which suggests stronger bonding. The partial densities of states (p-DOSs) for interstitial helium and its nearest iron atom were also checked to clarify why a higher charge transfer does not result in stronger bonding. Figure 3 indicates that the strongest interaction occurs between $\mathrm{Fe}-3 d$ and $\mathrm{He}-$ $2 s$. To further explore the spatial distribution of the interacting orbitals, the DOS was also plotted for orbitals with different magnetic quantum numbers. Two $e_{g}$ orbitals, $d_{z^{2}}$ and $d_{x^{2}-y^{2}}$, are found to have higher densities of states than 


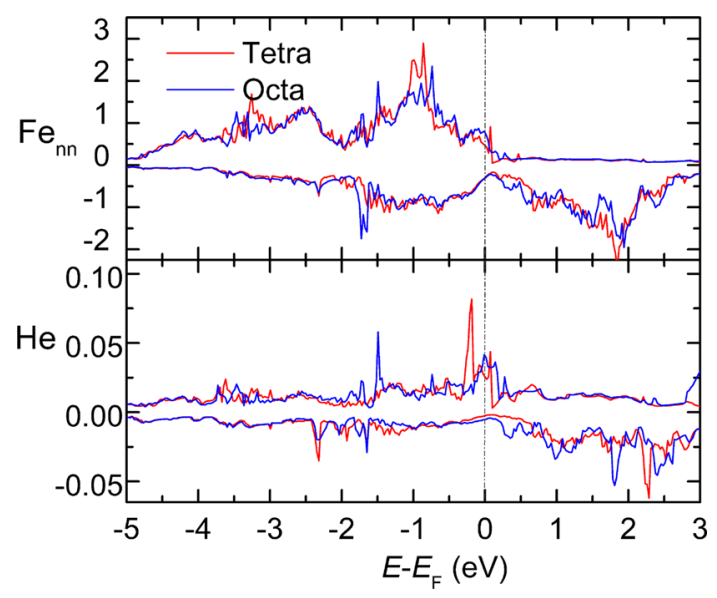

Fig. 2 Total densities of states for $\mathrm{Fe}$ and $\mathrm{He}$ atoms. The Fe atoms are at the nearest neighboring sites for both the octahedral and tetrahedral interstitials

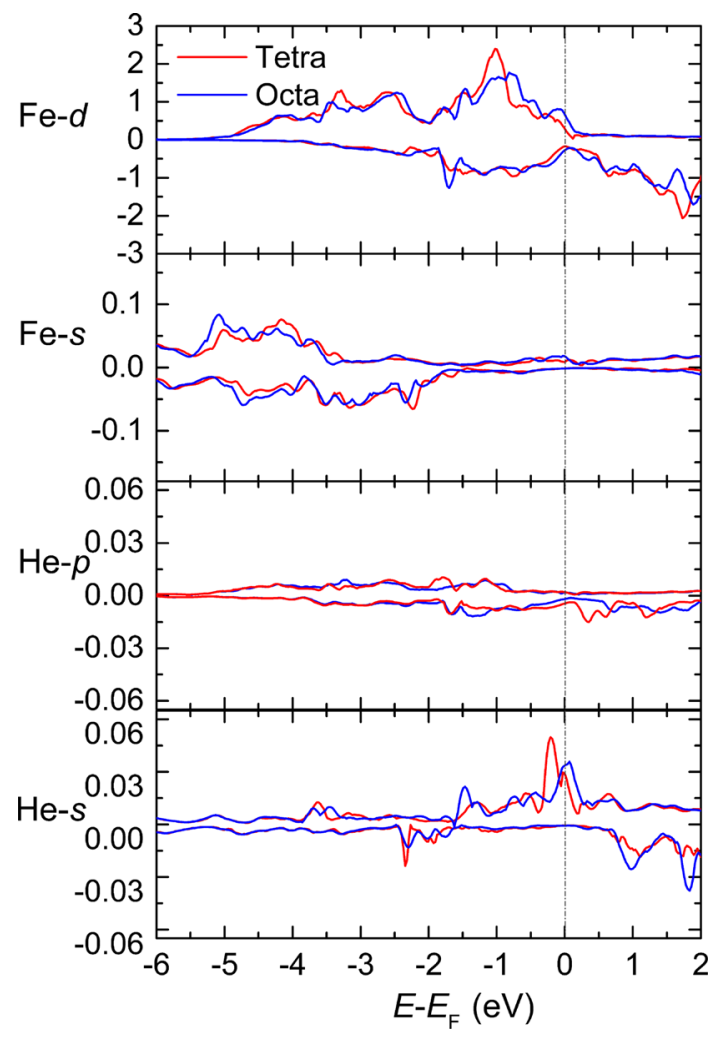

Fig. 3 Projected density of states over $\mathrm{Fe}$ and $\mathrm{He}$ atoms. The $\mathrm{Fe}$ atoms are at the nearest neighboring site for both octahedral and tetrahedral interstitials

the other extension directions of $\mathrm{Fe}-3 d$, so we mainly plot these two orbitals in Fig. 4. The DOS for the nearest iron atom redistributes among two $e_{g}$ orbitals because of the interstitial helium. Compared with the tetrahedral site, redistribution occurs more comprehensively for octahedral site, where the DOS of $d_{z^{2}}$ shows a drastic decrease while $d_{x^{2}-y^{2}}$ only shows a little decrease in the peak width. This

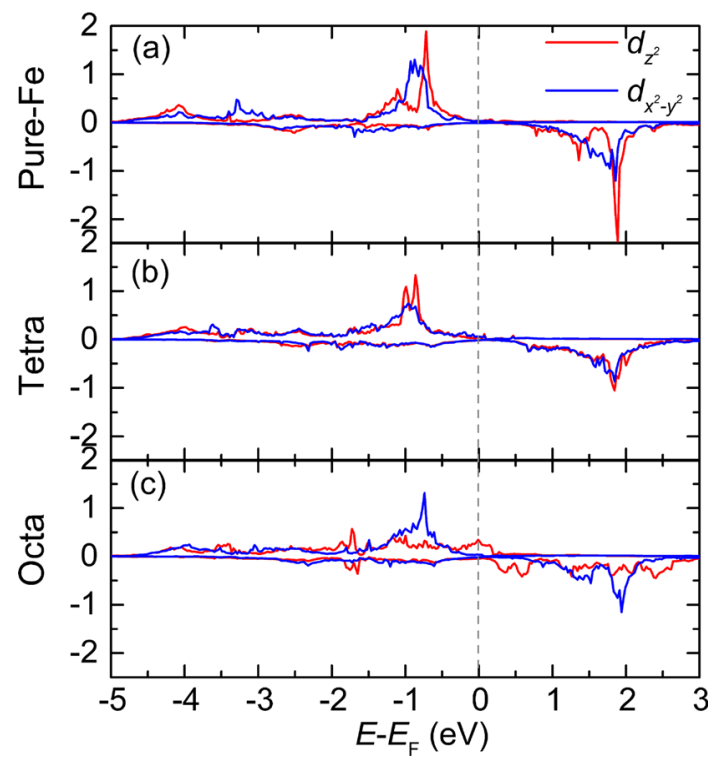

Fig. 4 Projected densities of states of $d_{x^{2}-y^{2}} / d_{z^{2}}$ orbitals for a atoms from pure $\mathrm{Fe}$; $\mathbf{b}$ the nearest neighboring atoms of the tetrahedral interstitial; $\mathbf{c}$ the nearest neighboring atoms of the octahedral interstitial

type of change in DOS can be connected with the special characters of the different orbitals. The $d_{z^{2}}$ orbital stretches along the $z$ direction, which has the closest distance between $\mathrm{Fe}_{\mathrm{nn}}$ and $\mathrm{He}$ at the octahedral site and along which most of the charge transfer happens. The decrease in the DOS in $d_{z^{2}}$ thus clearly weakens the bonding.

From the analysis above, it can be concluded that redistribution of DOS between the different orbitals and the symmetries of the sites is the key to explaining why more charge transfer does not result in stronger bonding. For an octahedral site, Fe- $d_{z^{2}}$ orbital should have the strongest interaction with He-s, but its DOS drops mostly. By contrast, for a tetrahedral site, the DOSs for different $\mathrm{Fe}$ orbitals are relatively average, which means that more orbitals are involved in the bonding. Additionally, tetrahedral sites have higher peaks in the DOS of helium and more neighboring atoms than octahedral sites. All of these can explain why a tetrahedral site has less charge transfer but stronger bonding, and thus a lower solution energy.

\subsection{Barrier of Migration}

To understand the bubbling behavior of helium, it is not enough to consider only the interaction between a helium atom and iron at an interstitial site, but it is also important to study the mobility of the helium between different interstitial sites. Migration barriers under different strains for different directions have been calculated by using CINEB. As tetrahedral sites are preferable for helium in bcc iron, the starting and ending states for the NEB calculation 
have been set to be two nearest neighboring tetrahedral sites. When no strain is applied, the migration barrier is $0.086 \mathrm{eV}$ for all the directions, which is similar to the value $0.06 \mathrm{eV}$ given by $\mathrm{Fu}$ et al. [20] using drag method in combination with DFT, and $0.078 \mathrm{eV}$ given by Morishita et al. [34] using Molecular Dynamics. Under uniaxial strain applied along the [100] $x$ axis, according to the symmetry of the strain tensor as well as that of the interstitial sites, there are three possible directions of migration that should be considered when uniaxial strain is applied, as shown in Fig. 5. The other directions are proven to be equivalent to these three directions by our simulation results.

The strain was first applied within the range of elastic strain from $0.2 \%$ compressive to $0.2 \%$ tensile for migration along the [011] direction, and the results are shown in Fig. 6a. It can be seen that the migration barrier follows a linear trend as the strain varies from $0.2 \%$ compressive to $0.2 \%$ tensile. When the strain is enlarged to $4 \%$, the migration barrier still follows the same linear trend. It is thus reasonable to use the results of a large strain to explicate the physical origins underlying the change of the migration barrier.

Strains varying from $4 \%$ compressive strain to $4 \%$ tensile strain are applied for all three migration directions, and their migration barriers follow different reverse trends, as shown in Fig. 6b. It is clearly seen that for the migration direction [101] (Fig. 5b) and [-110] (Fig. 5c), the migration barrier rises with the increase in tensile strain, but drop with increasing compressive strain; the trend is the reverse for direction [011] (Fig. 5a). The linearity of the trend suggests the possibility of explaining them using elastic theory, which has been successfully adopted by Zhou et al. [35] in their research regarding the solubility of hydrogen under strain.

To understand the differences underlying this unusual behavior, the differences in the stress symmetry of the starting, transition and ending states of the migration were studied. The lattice stress tensors are calculated and listed in Table 7, which are equivalent to the concept of a force dipole tensor [36]. The symmetries of the calculated force dipoles are in good agreement with the geometric symmetries of the occupation of helium, which can be deduced from the positions of the tetrahedral interstitials in Fig. 5 and the differential charge density distributions of the transition states in Fig. 7. The calculated stress tensors show good consistency for the different migration directions, and the same migration barrier $0.086 \mathrm{eV}$ under strain proves that three directions are equivalent under strain.

This symmetry is broken when strain is applied, and by using the stress tensor and the strain values, the variation of
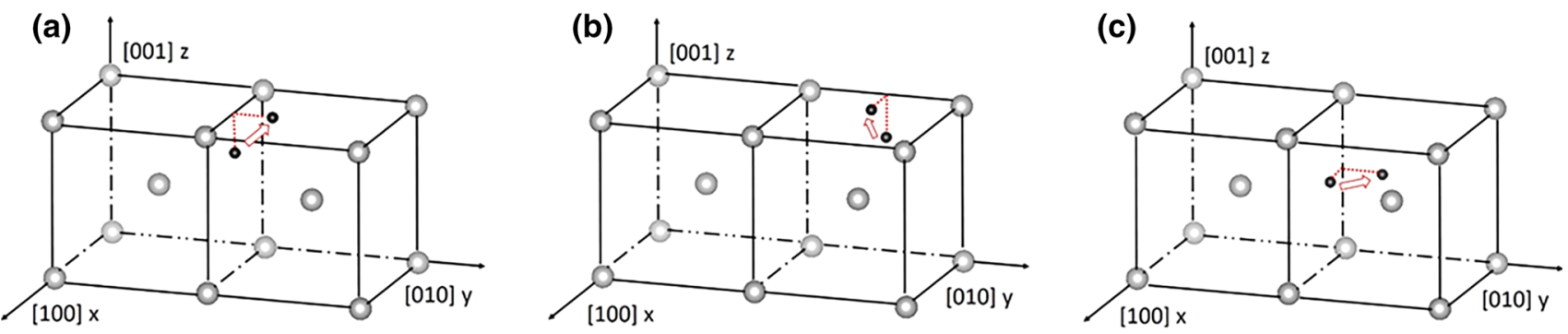

Fig. 5 Migration paths along different directions: a [011] $(\| y-z$ plane); b [101] $(\| x-z$ plane $) ; \mathbf{c}[-110](\| x-y$ plane $)$

(a)

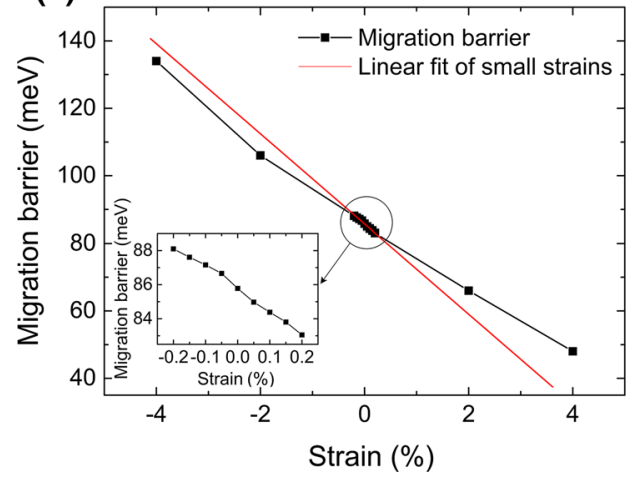

(b)

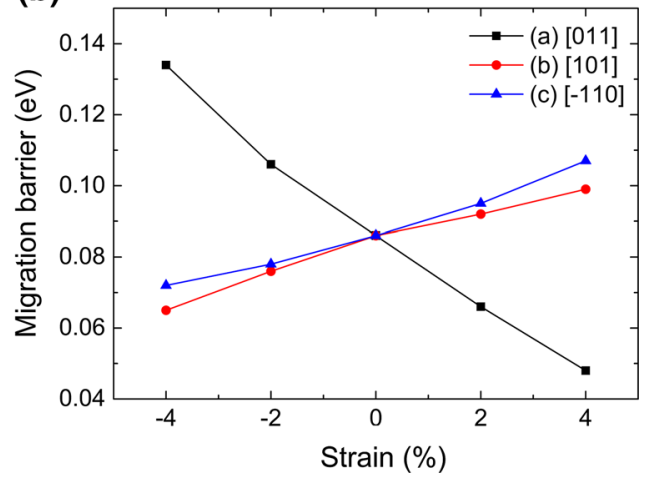

Fig. 6 a Migration barrier for direction [011] with data points of small strain ranging from $0.2 \%$ compressive to $0.2 \%$ tensile, b migration barrier as a function of the applied uniaxial strain for three different migration directions 
Table 7 Stress of the starting, transition and ending states for migration along different directions when no strain applied

\begin{tabular}{|c|c|c|c|c|c|c|c|}
\hline \multicolumn{2}{|l|}{ Direction } & \multicolumn{2}{|l|}{ Start } & \multicolumn{2}{|c|}{ Transition } & \multicolumn{2}{|l|}{ End } \\
\hline \multirow[t]{3}{*}{ [011] } & Direction & $x x(y y)$ & $z z$ & $y y(z z)$ & $x x$ & $z z(x x)$ & $y y$ \\
\hline & Stress (GPa) & -0.099 & -0.15 & 0.33 & -0.53 & -0.10 & -0.16 \\
\hline & $\sigma_{\Delta}(\mathrm{GPa})$ & -0.0015 & & -0.79 & & -0.0020 & \\
\hline \multirow[t]{3}{*}{ [101] } & Direction & $x x(y y)$ & $z z$ & $x x(z z)$ & $y y$ & $y y(z z)$ & $x x$ \\
\hline & Stress (GPa) & -0.096 & -0.15 & 0.34 & -0.54 & -0.10 & -0.15 \\
\hline & $\sigma_{\Delta}(\mathrm{GPa})$ & -0.00050 & & 0.42 & & -0.074 & \\
\hline \multirow[t]{3}{*}[-110]{} & Direction & $y y(z z)$ & $x x$ & $x x(y y)$ & $z z$ & $x x(z z)$ & $y y$ \\
\hline & Stress $(\mathrm{GPa})$ & -0.10 & -0.15 & 0.35 & -0.51 & -0.096 & -0.15 \\
\hline & $\sigma_{\Delta}(\mathrm{GPa})$ & -0.074 & & 0.41 & & -0.00050 & \\
\hline
\end{tabular}

Negative stands for compressive stress, calculated by VASP
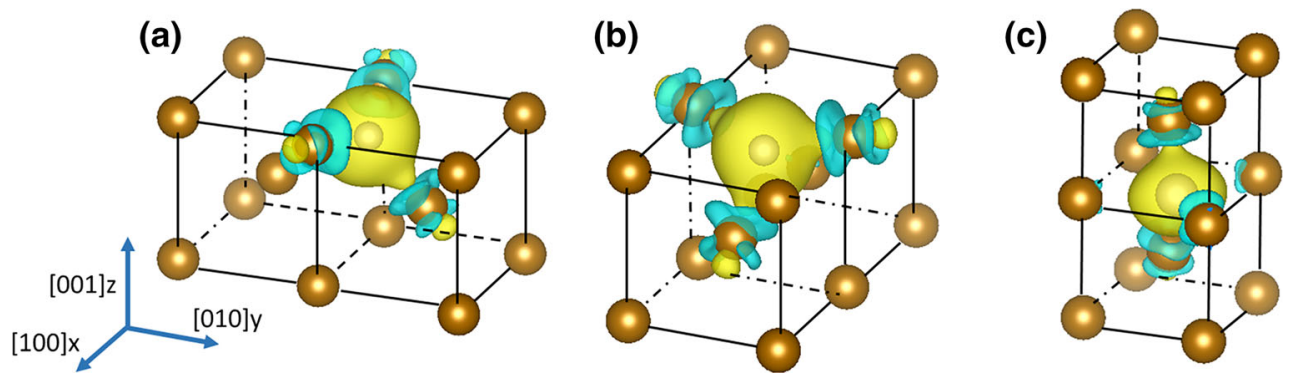

Fig. 7 Differential charge densities at the transition state for three different migration directions: a [011]; b [101]; c [-110]

the elastic energy of the starting and transition states can be expressed using elastic theory:

$$
\begin{aligned}
\Delta E & =V\left(\sigma_{x} \varepsilon_{x}+\sigma_{y} \varepsilon_{y}+\sigma_{z} \varepsilon_{z}\right)=V\left(\sigma_{x}-v \sigma_{y}-v \sigma_{z}\right) \varepsilon_{x} \\
& =V \sigma_{\Delta} \varepsilon_{x},
\end{aligned}
$$

$V$ is the volume of the supercell without strain, $\sigma_{x}, \sigma_{y}, \sigma_{z}$ are the stress tensors for the $x x, y y, z z$ directions, respectively, and $\varepsilon_{x}, \varepsilon_{y}, \varepsilon_{z}$ are the strains. The Poisson's ratio $v=0.39$ is also obtained from our calculation. For convenience of expression, we set a variable $\sigma_{\Delta}=\left(\sigma_{x}-v \sigma_{y}-v \sigma_{z}\right)$, and the variation of migration barrier under strain $\Delta E_{\text {mig }}^{\varepsilon}$ can then be expressed as:

$\Delta E_{\text {mig }}^{\varepsilon}=\Delta E^{\text {trans }}-\Delta E^{\text {start }}$,

$\Delta E^{\text {trans }}$ is the variation of the solution energy of helium at the transition state, and $\Delta E^{\text {start }}$ is that at the starting state.

Next, we elucidate how different symmetries reverse the variations of energy, using Eqs. (3) and (4) and data from Table 7. For the same strain $\varepsilon_{x}$, whether $\Delta E_{\text {mig }}^{\varepsilon}$ is positive or negative is determined by the values of $\sigma_{\Delta}$ of the transition states and starting states. Through our calculations, it is found that $\left|\Delta E^{\text {trans }}\right|$ is always higher than $\left|\Delta E^{\text {start }}\right|$ because of the higher absolute stress values at the transition states, so $\Delta E^{\text {trans }}$ can determine whether $\Delta E_{\text {mig }}^{\varepsilon}$ is positive or negative. Taking the compressive strain, for example, in which $\varepsilon_{x}$ will be negative, for the migration direction [011], $\sigma_{x}=-0.53 \mathrm{GPa}$, $\sigma_{y}=\sigma_{z}=0.33 \mathrm{GPa}$, and $\sigma_{\Delta}=-0.79 \mathrm{GPa}$, making $\Delta E^{\text {trans }}$ have a positive value, corresponding to the increase in the migration barrier under compressive strain. Meanwhile, for the migration direction [101] and [-110], because $\sigma_{x}$ has a positive value, $\sigma_{y}$ and $\sigma_{z}$ have opposite signs, $\sigma_{\Delta}=0.42 \mathrm{GPa}$ for [101] and $0.41 \mathrm{GPa}$ for [-110], making $\Delta E^{\text {trans }}$ have a negative value, which can explain the decrease in the migration barrier under tensile strain. Furthermore, for [101] and $[-110]$, although the migration energies have the same trend, there are small differences that can be attributed to the stress asymmetry of the starting states, with $\sigma_{\Delta}=-0.00050 \mathrm{GPa}$ for [101] and $-0.074 \mathrm{GPa}$ for [-110], making the migration barrier higher for [-110] under compressive strain. The situation is the reverse for the tensile strain.

The change in the elastic energy stems from the charge density difference during the migration. From the analysis above, it is indicated that a higher energy change occurs when the symmetry of the stress is the same as the symmetry of the strain, which is the case for the transition state of migration along [110] and the starting state of migration along [-110]. Figure 8 demonstrates how charge density will change at the transition state of migration along the [110] plane. Comparing Fig. 8c (4\% compressive strain) 
(a)

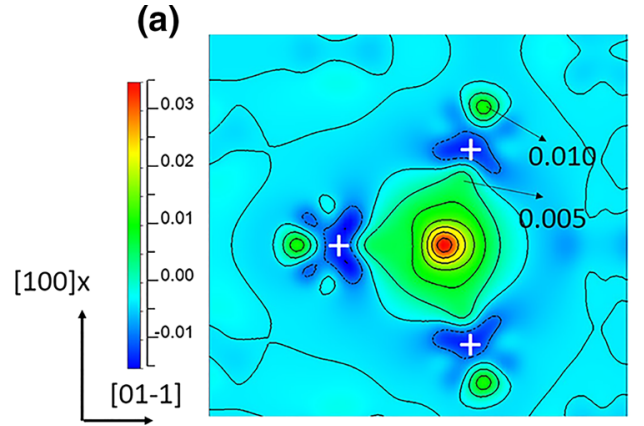

(b)

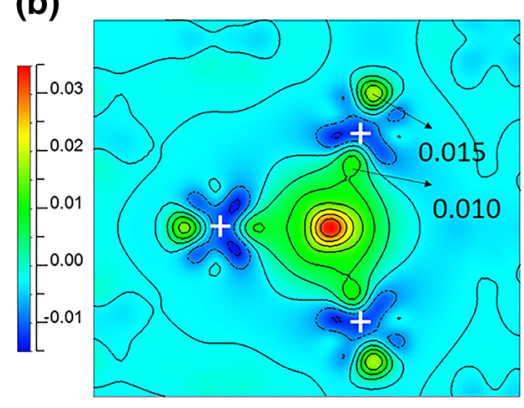

(c)

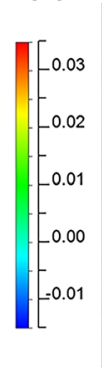

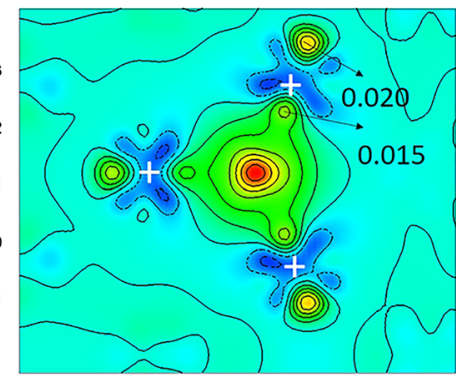

Fig. 8 Charge densities of the transition state of migration [110] under different strains (cross section, separated by 0.005 eV/ $\AA^{3}$ ): a $4 \%$ tensile strain, b no strain, c $4 \%$ compressive strain. The red and blue mean the highest and lowest charge density difference, respectively. The contour surfaces above 0 are shown by black solid lines, while contour surfaces below 0 are shown by black dashed lines (around the blue parts). The positions of the iron atoms are indicated with white crosses, and the helium lies in the center of the red part. (The difference of length in the $x$ direction is caused by the compressive strain applied)

with Fig. 8b (zero strain), we find that when a compressive strain is applied, the charge density exhibits an expansion of the blue zones between $\mathrm{Fe}$ and $\mathrm{He}$, which means that the charge density decreases, while the yellow zones that are labelled with numbers have an obvious charge increase. A greater charge transfer under compressive strain will lead to a higher electronic interaction energy of the transition state and increase the migration barrier. In contrast, the charge density under tensile strain of Fig. 8a shows less charge transfer than that in Fig. 8b, which leads to a lower migration barrier. For the migration paths [101] and [-110], the charge density of the transition states on the cross section is found to have not much change. The coupling effect of the electronic interaction energy and elastic strain energy together can well explain the trends of the migration barrier.

\section{Conclusions}

The solution and migration behaviors of $\mathrm{He}$ interstitials in bcc iron and their electronic origin have been studied by first principle calculation. Compared with the octahedral interstitial, the tetrahedral interstitial is found to have less charge transfer but stronger bonding and a lower solution energy. The physics for this behavior is revealed by a detailed analysis of DOS. For the octahedral site, the more uneven distribution of the DOS between different orbitals of $\mathrm{Fe}_{\mathrm{nn}}-3 d$ and the fewer neighboring atoms around the interstitial $\mathrm{He}$ atom makes the bonding weaker. Besides, the bonding between $\mathrm{Fe}$ and $\mathrm{He}$ is proved to be ionic by charge density difference, in which He gains electrons and $\mathrm{Fe}$ loses electrons. According to the energy band theory, the losing of valence electrons of the $\mathrm{Fe}$ atoms can weaken the metallic bonds, thus deteriorating the ductility and plasticity of the bcc iron.
The solution and migration behaviors under strain have been also studied. In a bcc iron lattice, helium tends to stay in the tetrahedral interstitial site, no matter whether a tensile or compressive strain is applied. The migration barrier, however, changes dramatically with the applied strain. With the variation of a uniaxial strain, the migration barriers of different directions follow different trends. In a strain-free lattice, the mobility of helium is isotropic, but with a strain applied, it is easier for the helium to migrate along particular directions than the others. This can break the random distribution of helium atoms in iron matrix and make it easier segregate into clusters, which facilitates the formation of helium bubbles under strain and can thus degrade the mechanical properties of bcc iron. In summary, our research analyzes the electronic structure change caused by the solution and migration of an interstitial helium in bcc iron with or without strain and offers possible explanations for how these initial microstructure processes can have an adverse effect on the mechanical properties. The conclusion can be linked with experimental results, as well as be used in simulations of larger scales such as MD, RT and FEA.

Acknowledgements This work was supported by the National Basic Research Programs of China (Nos. 2015GB118001, 2015CB654802, 20151080369 and 20141300796).

\section{References}

[1] H. Tanigawa, K. Shiba, A. Möslang, R.E. Stoller, R. Lindau, M.A. Sokolov, G.R. Odette, R.J. Kurtz, S. Jitsukawa, J. Nucl. Mater. 417, 9 (2011)

[2] J.F. Salavy, L.V. Boccaccini, P. Chaudhuri, S. Cho, M. Enoeda, L.M. Giancarli, R.J. Kurtz, T.Y. Luo, K. Bhanu Sankara Rao, C.P.C. Wong, Fusion Eng. Des. 85, 1896 (2010)

[3] R.J. Kurtz, A. Alamo, E. Lucon, Q. Huang, S. Jitsukawa, A. Kimura, R.L. Klueh, G.R. Odette, C. Petersen, M.A. Sokolov, P. Spätig, J.W. Rensman, J. Nucl. Mater. 386-388, 411 (2009) 
[4] W. Liu, C. Zhang, Z. Xia, Z. Yang, J. Nucl. Mater. 455, 402 (2014)

[5] M.R. Gilbert, S.L. Dudarev, S. Zheng, L.W. Packer, J.-Ch. Sublet, Nucl. Fusion 52, 083019 (2012)

[6] E.E. Bloom, J.T. Busby, C.E. Duty, P.J. Maziasz, T.E. McGreevy, B.E. Nelson, B.A. Pint, P.F. Tortorelli, S.J. Zinkle, J. Nucl. Mater. 367-370, 1 (2007)

[7] S.J. Zinkle, J.T. Busby, Mater. Today 12, 12 (2009)

[8] W. Liu, C. Zhang, Z. Yang, Z. Xia, Mater. Sci. Eng. A 583, 61 (2013)

[9] A. Hasegawa, M. Ejiri, S. Nogami, M. Ishiga, R. Kasada, A. Kimura, K. Abe, S. Jitsukawa, J. Nucl. Mater. 386, 241 (2009)

[10] R.E. Stoller, J. Nucl. Mater. 174, 289 (1990)

[11] B. Fu, B. Xu, W. Lai, Y. Yuan, H. Xu, C. Li, Y. Jia, W. Liu, J. Nucl. Mater. 441, 24 (2013)

[12] W. Liu, C. Zhang, Y. Ji, Z. Yang, H. Zang, T. Shen, L. Chen, Appl. Phys. Lett. 105, 121905 (2014)

[13] Z. Xia, C. Zhang, H. Lan, Z. Liu, Z. Yang, Mater. Lett. 65, 937 (2011)

[14] S.J. Zinkle, Fusion Sci. Technol. 64, 65 (2013)

[15] S. Jiang, F. Wan, Y. Long, J. He, P. Liu, S. Ohnuki, N. Hashimoto, Acta Metall. Sin. (Engl. Lett.) 26, 303 (2013)

[16] D.N. Braski, H. Schroeder, H. Ullmaier, J. Nucl. Mater. 83, 265 (1979)

[17] L. Mansur, W. Coghlan, J. Nucl. Mater. 119, 1 (1983)

[18] P. Trocellier, S. Miro, Y. Serruys, S. Vaubaillon, S. Pellegrino, S. Agarwal, S. Moll, L. Beck, Nucl. Instrum. Methods Phys. Res. B 331, 55 (2014)

[19] D. Stewart, Y. Osetskiy, R. Stoller, J. Nucl. Mater. 417, 1110 (2011)
[20] C.C. Fu, F. Willaime, Phys. Rev. B 72, 064117 (2005)

[21] C.S. Deo, M.A. Okuniewski, S.G. Srivilliputhur, S.A. Maloy, M.I. Baskes, M.R. James, J.F. Stubbins, J. Nucl. Mater. 361, 141 (2007)

[22] P. Zhang, T. Zou, J. Zhao, J. Nucl. Mater. 467, 465 (2015)

[23] T. Seletskaia, Y.N. Osetsky, R.E. Stoller, G.M. Stocks, J. Nucl. Mater. 351, 109 (2006)

[24] J. Cai, D. Lu, Acta Metall. Sin. (Engl. Lett.) 26, 25 (2013)

[25] C. Becquart, C. Domain, Phys. Rev. Lett. 97, 196402 (2006)

[26] P.E. Blöchl, Phys. Rev. B 50, 17953 (1994)

[27] G. Kresse, D. Joubert, Phys. Rev. B 59, 1758 (1999)

[28] J.P. Perdew, K. Burke, M. Ernzerhof, Phys. Rev. Lett. 77, 3865 (1996)

[29] W. Tang, E. Sanville, G. Henkelman, J. Phys. Condens. Matter 21, 084204 (2009)

[30] E. Sanville, S.D. Kenny, R. Smith, G. Henkelman, J. Comput. Chem. 28, 899 (2007)

[31] G. Henkelman, A. Arnaldsson, H. Jónsson, Comput. Mater. Sci. 36, 354 (2006)

[32] G. Henkelman, H. Jónsson, J. Chem. Phys. 113, 9978 (2000)

[33] G. Henkelman, B.P. Uberuaga, H. Jónsson, J. Chem. Phys. 113, 9901 (2000)

[34] K. Morishita, R. Sugano, B.D. Wirth, T. Diaz de la Rubia, Nucl. Instrum. Methods Phys. Res. Sect. B Beam Interact. Mater. Atoms 202, 76 (2003)

[35] H.B. Zhou, S. Jin, Y. Zhang, G.H. Lu, F. Liu, Phys. Rev. Lett. 109, 135502 (2012)

[36] C. Elsässer, M. Fähnle, L. Schimmele, C.T. Chan, K.M. Ho, Phys. Rev. B 50, 5155 (1994) 